\title{
Inkjet printed electronics using copper nanoparticle ink
}

\author{
Jin Sung Kang • Hak Sung Kim • Jongeun Ryu • \\ H. Thomas Hahn $\cdot$ Seonhee Jang $\cdot$ Jae Woo Joung
}

Received: 6 November 2009/Accepted: 20 December 2009/Published online: 8 January 2010

(C) The Author(s) 2010. This article is published with open access at Springerlink.com

\begin{abstract}
Inkjet printing of electrode using copper nanoparticle ink is presented. Electrode was printed on a flexible glass epoxy composite substrate using drop on demand piezoelectric dispenser and was sintered at $200{ }^{\circ} \mathrm{C}$ of low temperature in $\mathrm{N}_{2}$ gas condition. The printed electrodes were made with various widths and thickness. In order to control the thickness of the printed electrode, number of printing was varied. Resistivity of printed electrode was calculated from the cross-sectional area measured by a profilometer and resistance measured by a digital multimeter. Surface morphology of electrode was analyzed using scanning electron microscope (SEM) and atomic force microscope (AFM). From the study, it was found that 10 times printed electrode has the most stable grain structure and low resistivity of $36.7 \mathrm{n} \Omega \mathrm{m}$.
\end{abstract}

\section{Introduction}

In recent years, printed electronic technology has attracted lots of attentions in its applications such as flexible radio

\section{J. S. Kang $\cdot$ J. Ryu $\cdot$ H. Thomas Hahn}

Mechanical and Aerospace Engineering Department, University

of California, Los Angeles, CA 90095, USA

\section{H. S. Kim ( $\square)$}

Department of Mechanical Engineering, Hanyang University, Seoul, Republic of Korea

e-mail: kima1774@ucla.edu

H. Thomas Hahn

Korea Institute of Science and Technology, Seoul, South Korea

S. Jang · J. W. Joung

Samsung Electro-Mechanics, Suwon city, Gyunggi-do, South Korea frequency identification (RFID) tags [1], wearable electronics [2], organic light emitting diodes, and organic solar cells. Conventionally, mask-based photolithography method was used to make a desired conductive pattern. However, the subsequent processes of photolithography require etching and metal deposition such as solvent lift-off and electroplating process that accompany large amount of toxic chemical waste. Therefore, many researchers started to pay more attention on the direct inkjet printing method because it does not require the additional etching and metal deposition processes; it has only one-step printing procedure of making a desired conductive pattern on various substrates. In addition to the simple process, and cost and material reduction, the flexibility of changing patterns and the capability of printing on a large area are the most distinguishable advantages of printing method over the photolithography.

To use benefits of inkjet printing, fabrication conductive nanoink has become the most important issue. There are two general approaches in making a conductive ink [3]. The first method is to use solutions of metallic organic precursors, which can be reduced in low temperature. The second method, which is widely used in inkjet printing technology, is to use suspended metallic nanoparticles because they have low melting point than their bulk forms do [4].

Initially, suspension of gold and silver nanoparticles drew attention for a conductive ink fabrication because of their high conductivity and thermal stability. Bieri et al. [5] developed conductive ink by suspending gold nanoparticles in toluene, but it had high electrical resistivity of $140 \mathrm{n} \Omega \mathrm{m}$, which is 6 times larger than bulk gold does, and it had the problem of volatile organic compounds in industrial applications. In the work of Lee et al. [6], a conductive ink using silver nanoparticles was developed by 
dissociating silver nitrate in hydrocarbon using butylamine. However, both gold and silver are significantly more expensive than copper. Therefore, a conductive ink using copper nanoparticles drew more attention in recent years.

Several methods to fabricate a conductive ink using the copper nanoparticles were developed such as thermal decomposition, nonionic microemulsions, ultraviolet irradiation, and reduction of copper salts [7,8]. Yet, these developed processes were not economically efficient because of low throughput of less than $0.1 \mathrm{M}$. To resolve this problem, Lee et al. [9] introduced a chemically controlled fabrication process for copper nanoparticles; this novel process has high throughput of $0.2 \mathrm{M}$. In this study, we use the chemically controlled fabrication method to prepare the copper nanoink.

In addition to the high throughput fabrication of nanoinks, the characterization of reliability and property of the printed pattern is an essential step to the successful application of copper nanoink. As the nanoparticles in the ink are mixed with polymer binders and solvents, there are inevitable free spaces between the nanoparticles after the nanoink is dried. Moreover, the sintering process can induce cracks in the printed pattern because of the volume decrease by necking and fusion during the sintering process [8]. Therefore, it is essential to minimize those adverse effects in order to improve the reliability and quality of the printed electronics.

In this paper, we investigated the sintering phenomena of the copper nanoparticles and the effect of printed electrodes' thicknesses on the quality and reliability of the printed conductive patterns on a glass fiber/epoxy laminate. The surfaces of the electrodes were analyzed with SEM, profilometer, and AFM. X-ray tomography was used to investigate the cracks inside of the printed patterns.

\section{Experimental details}

\subsection{Specimen preparation}

The conductive copper nanoink for this study was prepared by Inkjet Business Group in Samsung Electro-Mechanics. To prepare the nanoink, copper nanoparticles were coated with the organic capping molecules, and then, it was dispersed in non-polar solvent at $40 \%$ by weight. The organic capping molecules were used to help disperse copper nanoparticles. Also, it was used to prevent aggregation of the copper nanoparticles at room temperature.

Using drop on demand piezoelectric inkjet with 128 nozzles, a designed circuit pattern was printed 5, 10, and 20 times to vary the thickness of the pattern. The printed patterns consist of $10 \mathrm{~cm}$ long line with two circular pads at the ends for measurements. Figure 1 shows the

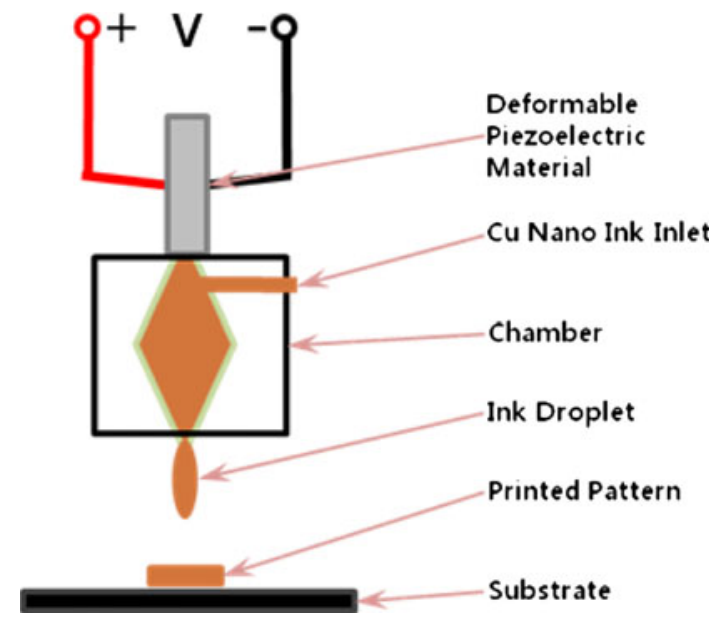

Fig. 1 Schematic drawing of drop-on-demand inkjet printing system

schematic diagram of drop on demand copper nanoparticle inkjet printer system, and Fig. 2 shows the printed electrode pattern. A woven glass fabric/bismaleimide triazine (BT) composite laminate of $100-\mu \mathrm{m}$ thickness was used as a printing substrate. BT core was kept at $85{ }^{\circ} \mathrm{C}$ to help solvent in the ink to be dried more quickly during the inkjet printing process.

The printed electrodes could be thermally sintered at low temperature of $200{ }^{\circ} \mathrm{C}$. The small particles can be sintered in low temperature because small particles have a higher proportion of surface atoms than large particles do, according to Schmidt et al. [10]. The surface atoms are less constrained in their thermal motion because they have fewer atoms in the neighbor than the atoms inside particles do. Therefore, as the proportion of the surface atom increases, a sintering temperature decreases. Also, Kang et al. [11] extended the explanation for low sintering temperature with the phonon softening: for copper nanoparticles, a mechanical energy required to cause a shear deformation is lower than its bulk form does. Optical microscope images of fully sintered printed electrodes on a BT core are shown on Fig. 3.

\subsection{Characterization}

The optical microscope (Olympus BX 41, Olympus, Japan) was used to analyze the sintered printed electrodes. The surface profile of each electrode was measured using a profilometer (Dektak 8 Surface Profiler, Veeco, USA). The AFM (Dimension 3100, Veeco, USA) was used for the nanoscale surface characterization. The SEM (JSM-6700F, JEOL, Japan) were used to analyze surface cracks and the surface morphology of sintered copper nanoparticles. The X-ray tomography (SkyScan 1172, SkyScan, Belgium) was used for three-dimensional microscale surface characterization. Four-point probes method was used to measure the 


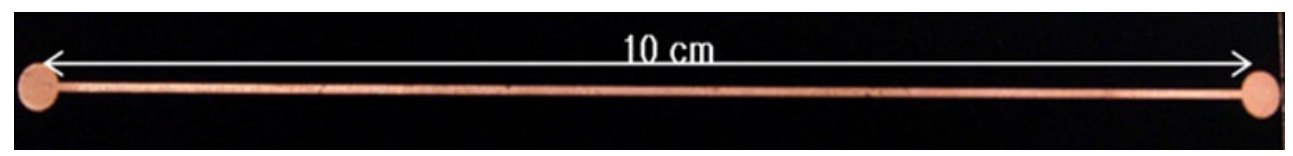

Fig. 2 Printed electrode
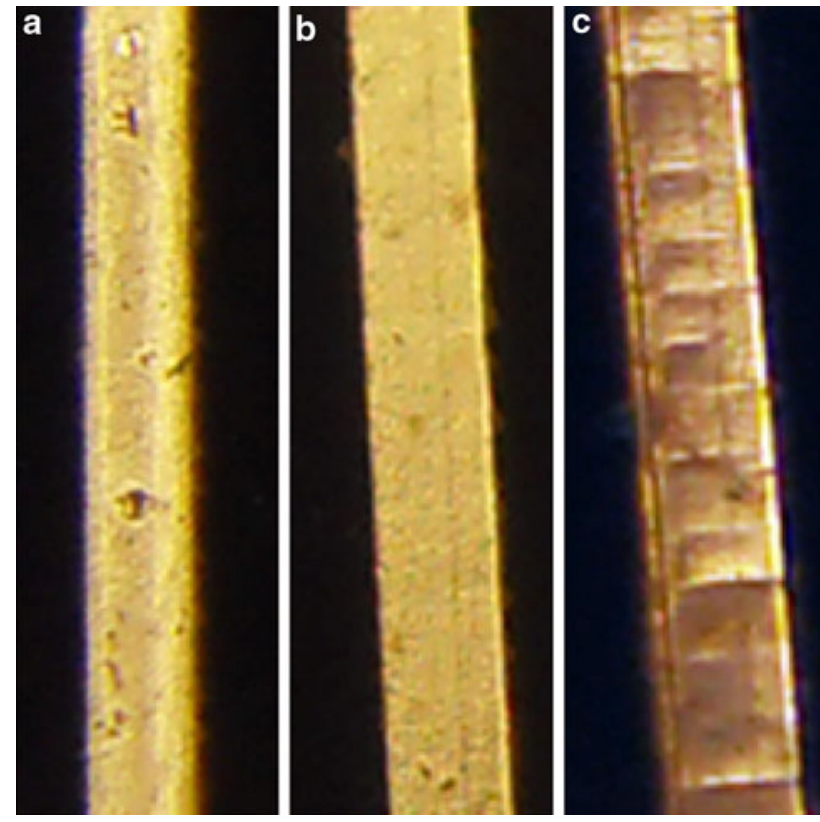

Fig. 3 Optical microscope images of fully sintered printed electrodes: a 5 times printed, b 10 times printed, and c 20 times printed

resistance of each printed electrode using a digital multimeter (Keithley 2100, Keithley Instruments, USA). An electrical resistivity was calculated using following equation:

$\rho=\frac{R A_{\mathrm{c}}}{L}$

where $R$ is the electrical resistance, $A_{\mathrm{c}}$ is the cross-sectional area, $L$ is the length, and $\rho$ is the electrical resistivity of an printed electrode.

\section{Results and discussions}

According to the literatures, the melting temperature of metallic nanoparticles was calculated by the following equation [12]:

$T_{\mathrm{m}}=T_{0} \times\left(1-\frac{2}{\rho_{\mathrm{s}} L r}\left[\gamma_{\mathrm{s}}-\gamma_{1}\left(\frac{\rho_{\mathrm{s}}}{\rho_{\mathrm{l}}}\right)^{2 / 3}\right]\right)$

where $\rho$ is the density, $\gamma$ is the surface tension, $T_{0}$ is the melting temperature of bulk metal, $L$ is the latent heat of fusion, and $r$ is the radius of the nanoparticle. The subscripts $\mathrm{s}$ and 1 denote solid and liquid, respectively. The material constants of copper are shown in Table 1; the liquid state properties are taken from copper at the temperature of 1,500 K. Equation (2) predicts that the melting temperature of copper is significantly depressed much lower than $600{ }^{\circ} \mathrm{C}$ when the particle radius is smaller than $10 \mathrm{~nm}$. Also, because sintering temperature is defined as a temperature at which the grains of solid formed from small particles connecting at its surfaces, sintering temperatures are $20 \%$ lower than melting temperature for a copper, according to Mackenzie and Shuttleworth [13]. In this study, copper nanoparticles were fully sintered at $200{ }^{\circ} \mathrm{C}$ in inert atmosphere, nitrogen gas to prevent the oxidation of copper nanoparticles.

Figure 4 shows the sintering result of the copper nanoparticles ink as function of sintering time for 5 times printed electrode. It was found that the copper nanoparticles could be sintered fully in $20 \mathrm{~min}$. Figure 5 shows high resolution SEM images of inkjet printed electrode before and after $20 \mathrm{~min}$ thermal sintering; dispersed copper nanoparticles were agglomerated and sintered after thermal sintering.

Profilometer results are shown on Table 2. Average thicknesses of 5, 10, and 20 times printed electrodes were $1,731,3,690$, and $11,954 \mathrm{~nm}$, respectively. Surface profiles of printed electrodes using a profilometer are shown in Fig. 6. The roughness calculated from the profilometer result were $1,100,500$, and $3,000 \mathrm{~nm}$ for 5,10 , and 20 times printed electrodes, respectively. Therefore, 10 times printed electrodes had more uniform surface profile than other thickness cases.

Based on the thicknesses and widths measured by profilometer, average resistivity of 5 times printed, 10 times printed, and 20 time printed electrodes were calculated to be $61.3,36.7$, and $98.9 \mathrm{n} \Omega \mathrm{m}$, respectively. Resistivities of each printed electrodes are presented on Table 2 and Fig. 7. It was found that the 10 times printed electrode has the lowest electrical resistivity.

At low SEM magnification of 1,000 times (Fig. 8), many small pre-existing surface cracks were found on the

Table 1 Physical constants of copper

\begin{tabular}{lll}
\hline & Solid & Liquid \\
\hline Specific gravity, $\rho\left(\mathrm{kg} \mathrm{m}^{-3}\right)$ & 8,960 & 7,998 \\
Surface tension, $\gamma\left(\mathrm{J} \mathrm{m}^{-2}\right)$ & 1.8 & 1.1 \\
Latent heat of fusion, $L\left(\mathrm{~J} \mathrm{~kg}^{-1}\right)$ & $2.05 \times 10^{5}$ & - \\
\hline
\end{tabular}


Fig. 4 Sintering of the copper nanoparticles ink as function of sintering time

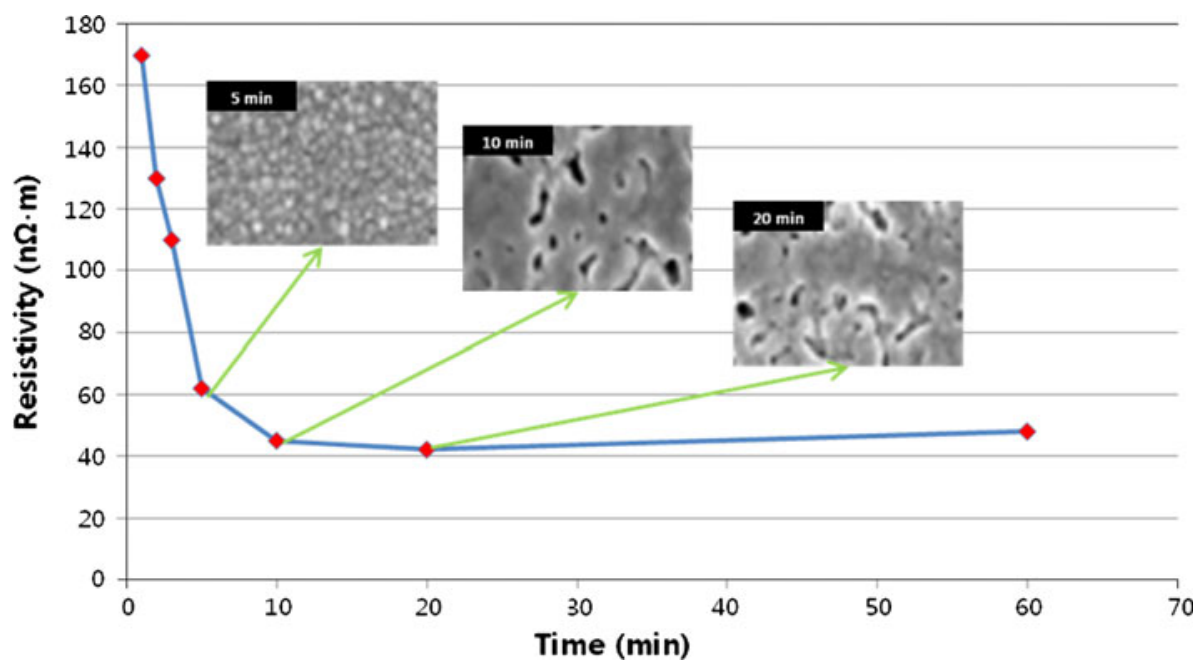

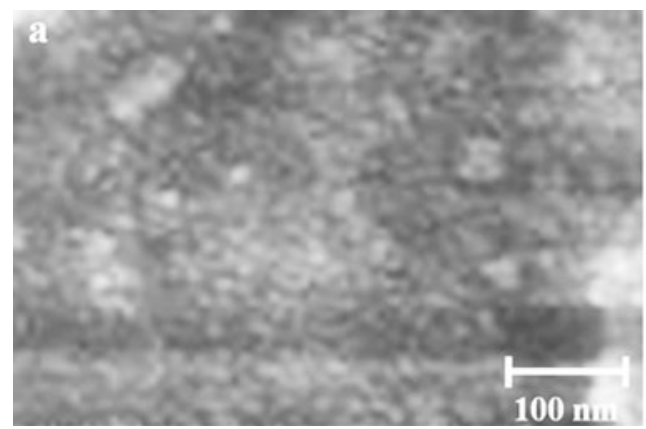

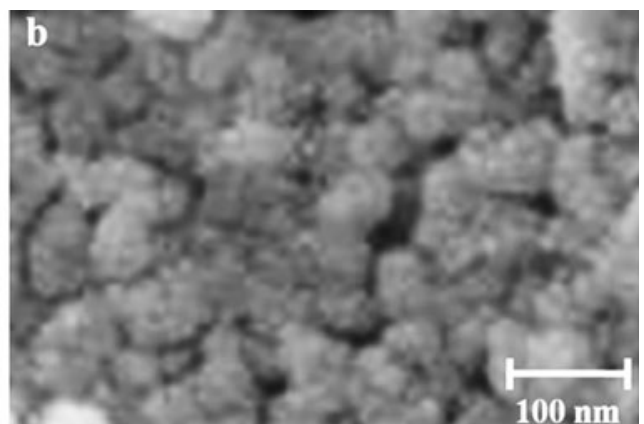

Fig. 5 SEM images of inkjet printed electrode $\mathbf{a}$ before, and $\mathbf{b}$ after thermal sintering

Table 2 Dimensions and electrical resistance of printed electrodes

\begin{tabular}{lccccc}
\hline \# of printing & Resistance $(\Omega)$ & Width $(\mu \mathrm{m})$ & $\begin{array}{l}\text { Average } \\
\text { thickness }(\mathrm{nm})\end{array}$ & Length $(\mathrm{cm})$ & $\begin{array}{l}\text { Resistivity } \\
(\mathrm{n} \Omega \mathrm{m})\end{array}$ \\
\hline 5 & 11.2882 & 409.68 & 1,979 & 10 & 91.497 \\
& 6.4898 & 471.73 & 1,480 & 10 & 45.306 \\
& 4.7632 & 569.04 & 1,736 & 10 & 47.051 \\
10 & 2.6414 & 368.01 & 3,736 & 10 & 36.316 \\
& 2.4143 & 398.93 & 3,773 & 10 & 36.339 \\
& 1.9327 & 564.38 & 3,255 & 10 & 35.505 \\
& 1.5616 & 621.08 & 3,996 & 10 & 38.757 \\
& 1.4849 & 410.27 & 13,295 & 10 & 80.993 \\
& 1.4231 & 494.33 & 10,926 & 10 & 76.861 \\
& 1.3917 & 560.37 & 11,053 & 10 & 86.199 \\
& 1.8719 & 645.10 & 12,543 & 10 & 151.467 \\
\hline
\end{tabular}

surface of 5 times printed electrode while the large cracks were found in the 20 times printed electrode. However, 10 times printed electrode does not show any severe surface cracks (Fig. 8b). The mechanism of this pre-existing small surface crack generation can be explained as follows. Preexisting surface cracks in printed electrodes were caused during sintering of copper nanoparticle: copper nanoparticles are randomly sintered with either left of right copper nanoparticle because smaller particle melts faster, and the lengths of sintered copper particles were smaller than lengths of unsintered copper particles, according to Zhu [14]. Schematic of this sintering process with crack propagation is shown in Fig. 9. Moreover, according to Scherer and Garino [15], when nanoparticles are sintered on a rigid substrate, shrinkage is inhibited on a plane of the substrate; therefore, tensile stress arises through the thickness of the layer, which may promote more the small crack generation during the sintering process. Therefore, it can be concluded 

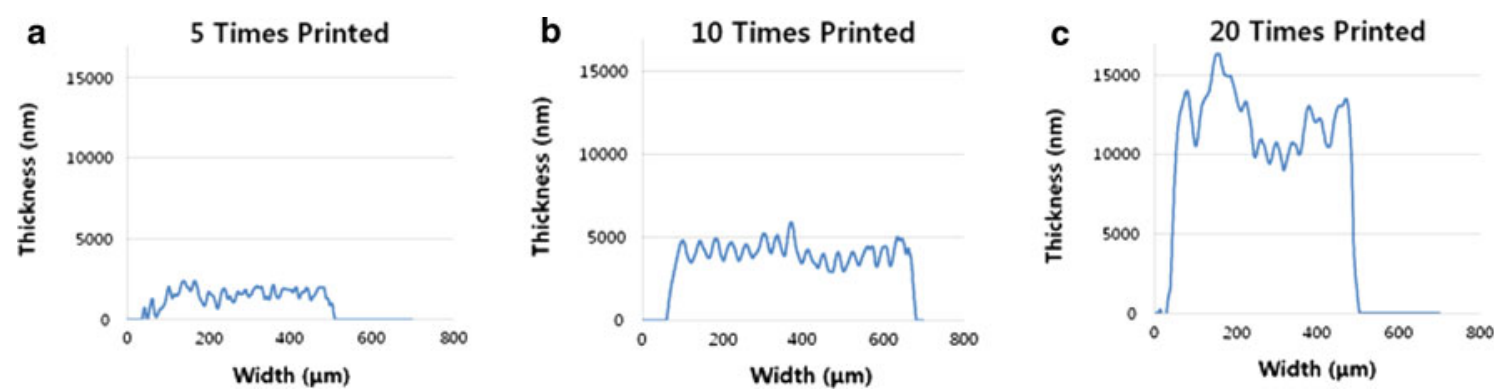

Fig. 6 Surface profiles of printed electrode using profilometer: a 5 times printed, b 10 times printed, and c 20 times printed

Fig. 7 Resistivity of printed electrodes depended on their widths and number of printing compared to resistivity of bulk copper
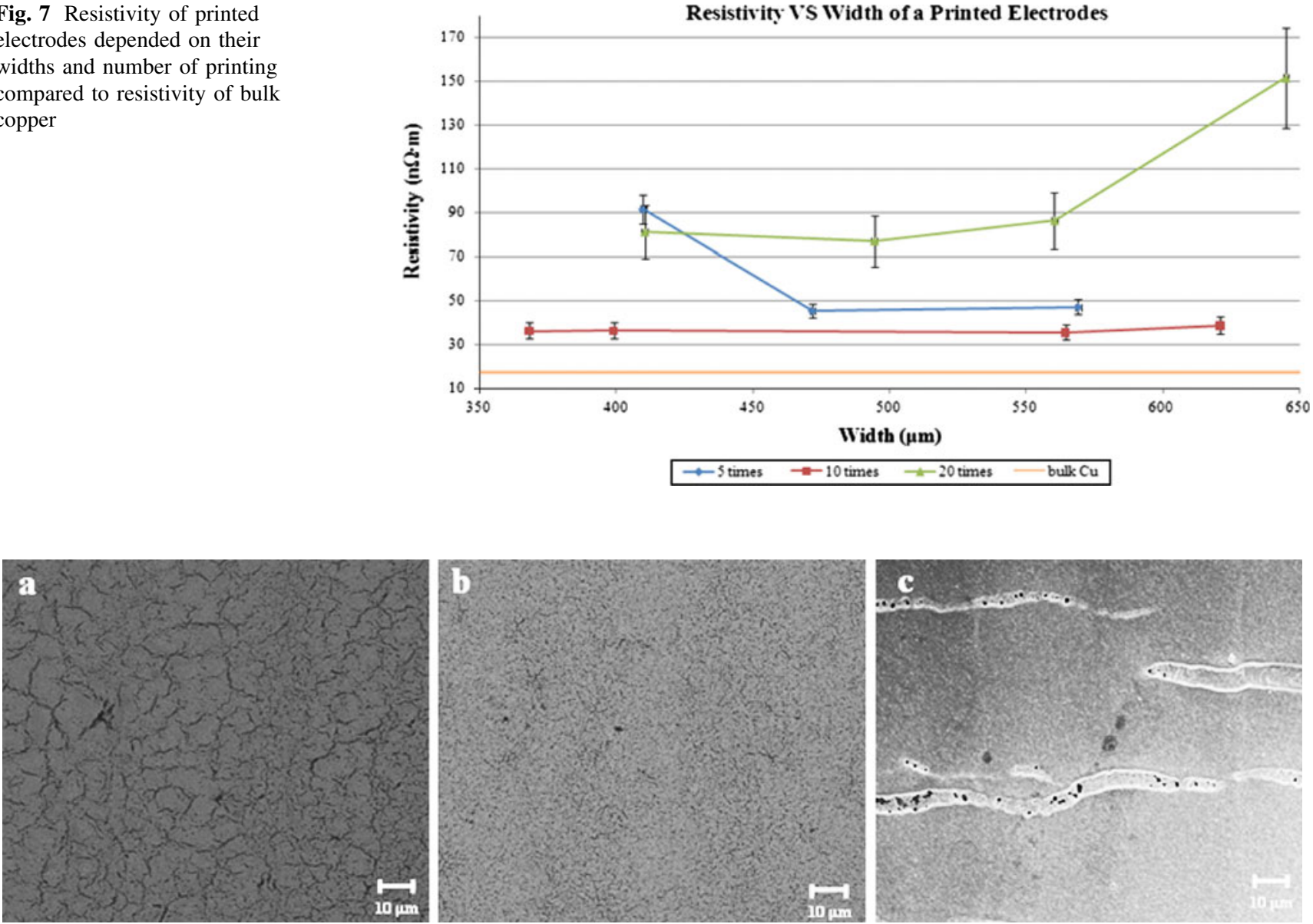

Fig. 8 SEM images of printed electrodes at 1,000 times magnification: a 5 times printed $\mathbf{b} 10$ times printed, and $\mathbf{c} 20$ times printed

that range of particle size should be reduced as much as possible to reduce the amount of pre-existing cracks.

As a proof of pre-existing cracks, three-dimensional surface profiles of printed electrode were taken on $1 \mu \mathrm{m} \times 1 \mu \mathrm{m}$ square using AFM. In Fig. 10, lines on AFM images of printed electrodes indicate the pre-existing cracks. All 5, 10, and 20 times printed electrodes have preexisting cracks on their surface. As Fig. 8 shows, 10 times printed electrode's cracks were not observable because 10 times printed electrode had enough number of printed layers to cover the pre-existing cracks on the bottom layers (Fig. 11b). Unlike 10 times printed electrode, 5 times printed electrode does not have enough layers to cover preexisting cracks; therefore, cracks are shown on SEM images of 1,000 times magnification (Fig. 8).

Using SEM magnification of 50,000 times, agglomerations of copper nanoparticles were observed for 5, 10, and 20 times printed electrodes, shown in Fig. 12a-c, respectively. Voids in 5 times printed electrodes were bigger than 10 times printed electrodes. Similarly, voids in 10 times 
Fig. 9 Schematic drawing of producing preexisting cracks
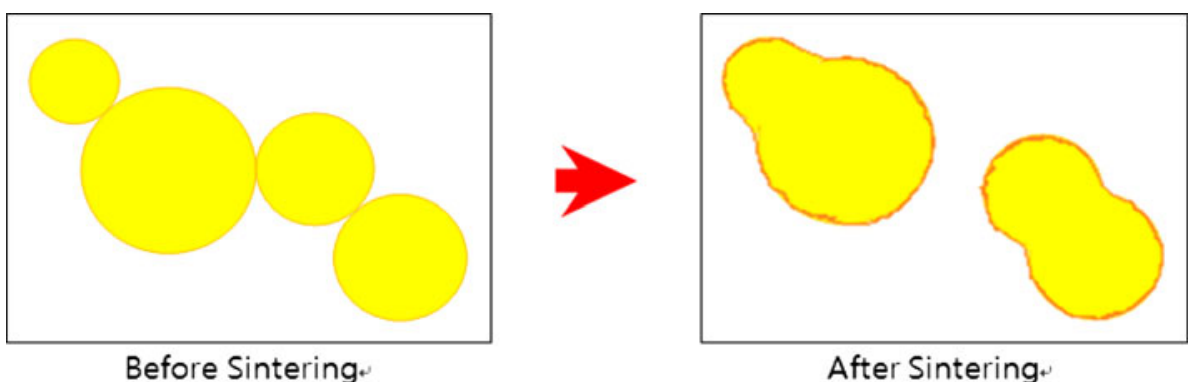

After Sintering. a

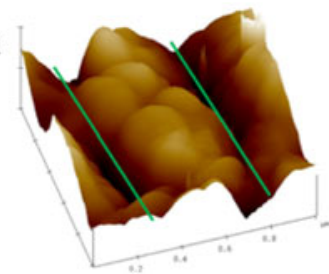

b

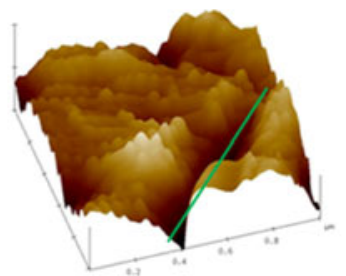

C

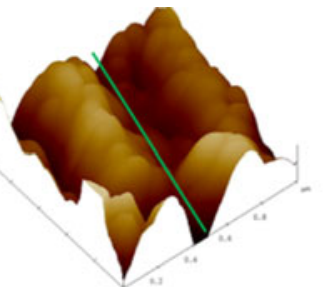

$\times 0.200 \mathrm{~mm} / \mathrm{div}$

z $28.000 \mathrm{~nm} / \mathrm{div}$

Fig. 10 AFM images of printed electrodes with green lines passing through preexisting cracks on their top surfaces: a 5 times printed, b 10 times printed, and c 20 times printed

Fig. 11 Schematic drawing of a producing preexisting cracks in 5 times printed electrode, b covering preexisting cracks in 10 times printed electrode, and $\mathbf{c}$ crack propagation in 20 times printed electrode

a
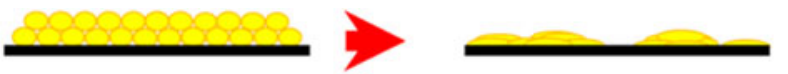

Not enough layers to cover cracks

b
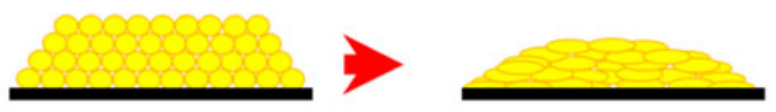

Enough layers to cover cracks

C
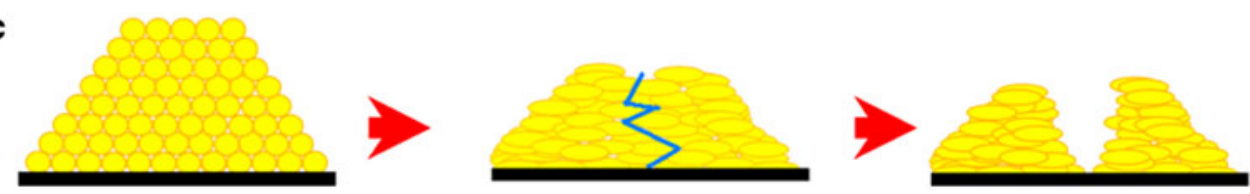

Thickness is probably greater than critical thickness. causing preexisting cracks to grow.
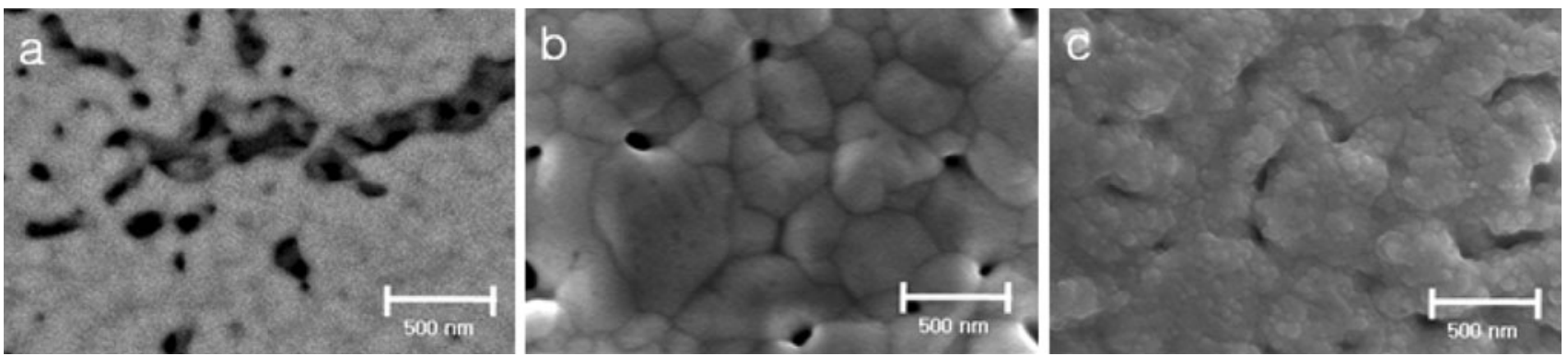

Fig. 12 SEM images of printed electrodes at 50,000 times magnification: a 5 times printed, b 10 times printed, and c 20 times printed

printed electrodes were bigger than voids in 20 times printed electrode, showing that copper nanoparticles were most densely sintered for 20 times printed electrodes.

Although 20 times printed electrode was more densely agglomerated, and had more number of layers than 10 times printed one did, severe microscopic cracks were observed at low SEM magnification (Fig. 8) and X-ray tomography (Fig. 13). Small cracks on 5 times printed electrode was not observed using X-ray tomography because of its low resolution. Figure $8 \mathrm{c}$ shows that cracks on 20 times printed electrode grew into larger cracks. Reasons for existence of these large cracks can be explained by following two reasons. First, according to Scherer and Garino [15], less porous film experience more 
Fig. 13 Surface profiles of printed electrode using X-ray tomography: a 5 times printed, b 10 times printed, and c 20 times printed
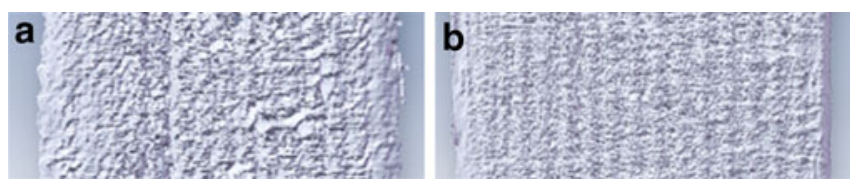

stress from a rigid substrate. Since 20 times printed electrode is the least porous, it experienced highest stress from its rigid substrate.

Secondly, according to Bordia and Jagota [16], crack grows when the thickness of the film is greater than the critical thickness. The governing equation for crack growth in the sintered thin film can be expressed as follows:

$\sqrt{\frac{1}{k R}>\sqrt{\frac{a}{R}\left(\frac{\pi}{1-v^{2}}\right)}}$

where $k$ is a friction parameter, $a$ is a half of crack length, $R$ is a distance from a crack tip to the center of the first contact, and $v$ is Poisson's ratio. The friction parameter controls the crack growth, which depends on the film thickness. According to Jagota and Hui [17], the friction parameter can be expressed as below,

$k=\sqrt{\frac{(1-v)}{\frac{2}{3} t^{2}+\frac{4}{3} t}}$

where $t$ is the film thickness. Since $k$ varies inversely with $t$, the friction parameter decreases as the film thickness increase. In Eq. (3), as $\mathrm{k}$ decrease, $\sqrt{1 /(k R)}$ increase, this will result in the crack growth. Critical thickness is a thickness of sintered film that results in the growth of preexisting cracks. Therefore, it can be inferred that 20 times printed electrode has thickness of $3,690 \mathrm{~nm}$, that is larger than its critical thickness, while those of 5 and 10 times printed electrodes do not exceed the critical thickness.

Lastly, even though 10 times printed electrode has the best quality, the lowest resistivity, its resistivity is higher than that of bulk copper, $17.2 \mathrm{n} \Omega \mathrm{m}$. Higher resistivity might come from the micro/nano porous structure of the sintered copper electrode as shown in Fig. 9. However, this resistivity of the printed electrode is suitable to be used in industrial applications such as RFID tags because our printed electrodes' resistivity is lower than $87 \mathrm{n} \Omega \mathrm{m}$, which is the appropriate resistivity value for RFID application, [18].

\section{Conclusions}

Inkjet printed electrodes with various dimensions were characterized. Specific results are

1. To reduce the amount of pre-existing cracks, range of particle size should be reduced as much as possible because the pre-existing cracks on surface are mainly caused by having wide range of particle sizes.

2. To have good quality of printed electrode, number of printing should not be large because when the thickness become larger than its critical thickness, printed electrodes' pre-existing cracks grow into large cracks.

3. Resistivity of printed electrode using our copper nanoparticles was $36.7 \mathrm{n} \Omega \mathrm{m}$, which is suitable for industrial applications such as RFID.

Acknowledgment The authors would like to thank the Air Force Office of Scientific Research for supporting us through a MURI grant FA 9550-06-1-0326 to the University of Washington. Also, the authors are grateful to Dr. Les Lee for his encouragements toward this work. Lastly, the authors appreciate Samsung Electro-Mechanics for providing printed electronics using copper nanoparticles.

Open Access This article is distributed under the terms of the Creative Commons Attribution Noncommercial License which permits any noncommercial use, distribution, and reproduction in any medium, provided the original author(s) and source are credited.

\section{References}

1. S.K. Volkman, Y. Pei, D. Redinger, S. Yin, V. Subramanian, Mater. Res. Soc. Symp. Proc. 814, 151-156 (2004)

2. S.M. Bidoki, D. McGorman, D.M. Lewis, M. Clark, G. Horler, R.E. Miles, AATCC Rev 5, 11 (2005)

3. D. Kim, J. Moon, Electrochem. Solid-State Lett. 8, J30 (2005)

4. G.L. Allen, R.A. Bayles, W.W. Gile, W.A. Jesser, Thin Solid Films 144, 297-308 (1986)

5. N.R. Bieri, J. Chung, S.E. Haferl, D. Poulikakos, C.P. Grigoropoulos, Appl. Phys. Lett. 82, 3529 (2003)

6. K.J. Lee, B.H. Jun, T.H. Kim, J. Joung, Nanotechnology 17, 2424 (2006)

7. M. Aslam, G. Gopakumar, T. Shoba, I. Mulla, K. Vijayamohanan, S. Kulkarni, J. Urban, W. Vogel, J. Colloid Interface Sci. 255, 79-90 (2002)

8. L. Qi, J. Ma, J. Shen, J. Colloid Interface Sci. 186, 498-500 (1997)

9. Y. Lee, J. Choi, K.J. Lee, N.E. Stott, D. Kim, Nanotechnology 19, 415604 (2008)

10. M. Schmidt, R. Kusche, B. von Issendorff, H. Haberland, Nature 393, 21 (1998)

11. J.W. Kang, H.J. Hwang, J Korean Phys Soc 40, 946-948 (2002)

12. P. Buffat, J.P. Borel, Phys. Rev. A Gen. Phys. 13, 2287-2298 (1976)

13. J.K. Mackenzie, R. Shuttleworth, Proc. Phys. Soc. Sect. B 62, 833-852 (1949)

14. H. Zhu, Philos. Mag. Lett. 73, 27-33 (1996) 
15. G.W. Scherer, T. Garino, J. Am. Ceram. Soc. 68, 216-220 (1985)

16. R.K. Bordia, A. Jagota, J. Am. Ceram. Soc. 76, 2475-2485 (1993)

17. A. Jagota, C. Hui, Mech. Mater. 9, 107-119 (1990)
18. V. Subramanian, P. Chang, D. Huang, J. Lee, S. Molesa, D. Redinger, S. Volkman, VLSI Design held jointly with 5th international conference on embedded systems and design., 19th international conference on, 6 (2006) 